\title{
Traumatic Optic Neuropathy
}

\author{
Sun Young Jang \\ Department of Ophthalmology, Soonchunhyang University Bucheon Hospital, Soonchunhyang University College of Medicine, \\ Bucheon, Korea
}

Traumatic optic neuropathy (TON) refers to optic nerve injury resulting from direct and indirect head and facial trauma. The pathogenesis of indirect TON has not been fully elucidated, and the management of TON remains controversial. In this review article, I review the recent literature regarding TON and discuss how to manage indirect TON.

(Korean J Neurotrauma 2018;14(1):1-5)

KEY WORDS: Direct · Indirect · Optic nerve · Trauma · Treatment.

\section{Introduction}

Traumatic optic neuropathy (TON) is rare, but can cause severe, irreversible vision loss. Since Hippocrates first noted visual impairment after facial trauma, ${ }^{11)}$ numerous reports have described TON, focusing on its causes and methods of treatment. ${ }^{1,714-16,24-26)}$ In this article, I review the recent literature regarding TON and discuss how to manage indirect TON.

\section{Classification of TON}

TON can be classified as direct and indirect TON, based on the mechanism of causative injury. ${ }^{20,25)}$ When projectile or sharp objects injure the optic nerve directly, these cases are diagnosed as direct TON. ${ }^{25)}$ Thus, direct TON is characterized by the presence of an open wound, leading to direct injury of the optic nerve, and can be diagnosed by neuroimaging, such as computed tomography or magnetic resonance

Received: February 26, 2018 / Revised: April 3, 2018

Accepted: April 5, 2018

Address for correspondence: Sun Young Jang

Department of Ophthalmology, Soonchunhyang University Bucheon Hospital, Soonchunhyang University College of Medicine, 170 Jomaru-ro, Bucheon 14584, Korea

Tel: +82-32-621-6718, Fax: +82-32-621-5018

E-mail: ysyat01@naver.com

$@$ This is an Open Access article distributed under the terms of Creative Attributions Non-Commercial License (http://creativecommons. org/licenses/by-nc/4.0/) which permits unrestricted noncommercial use, distribution, and reproduction in any medium, provided the original work is properly cited. imaging. Direct TON is extremely rare because of the protection provided by the bony orbit.

In 2004 , Sarkies ${ }^{20)}$ provided an update on the several varieties of direct TON in a review article. The author included optic nerve avulsion, optic nerve sheath hemorrhage, and orbital hemorrhage, as well as orbital emphysema, as direct TON disease entities. Such varieties of direct TON can be recognized ophthalmoscopically or with another imaging technique, such as ultrasonography. ${ }^{20,21)}$ For example, optic nerve avulsion results from rotation of the eyeball and/ or raised intraocular pressure. This means that an open wound is not always necessary to diagnose direct TON. In optic nerve avulsion, the optic nerve is forcibly disinserted from the eyeball and the lamina cribrosa is retracted from the scleral rim. ${ }^{18,21)}$

Although several varieties of optic nerve diseases have been added to the list of direct TON entities, indirect TON remains more common than direct TON. Indirect TON results from concussive force to the head, particularly the forehead. The prevalence of indirect TON in closed-head trauma has been reported as $0.5 \%$ to $5 \% .^{20,25)}$ The site of injury causing blindness is typically the forehead or supraorbital ridge, and less commonly may be the temporal region. ${ }^{20,23)}$

Recently, Magarakis et al. ${ }^{19)}$ systematically reviewed the literature to identify specific facial bone fracture patterns associated with ocular injury, including blindness. The authors found that zygomatic-maxillary complex fractures were the most common type of injury complicated by loss 
of vision. According to a study by Ansari ${ }^{2}$, in which a total of 2,503 patients were enrolled, retrobulbar hemorrhage was thought to be the primary mechanism of blindness, followed by direct damage to the eyeball. Laceration and compression of the optic nerve comprised $10 \%$ and $6.6 \%$ of visual loss, respectively. Dancey et al. ${ }^{5)}$ showed that the primary mechanism of blindness after blunt facial trauma was TON ( $n=12)$, optic nerve compression $(n=3)$, and globe rupture $(n=2)$, in an investigation of 2,400 patients with facial fractures.

\section{Pathophysiology of Indirect TON}

Although the mechanism of indirect TON is not fully understood, the cellular and biochemical pathophysiology of brain and spinal cord trauma provide insight into mechanisms that may operate in TON. The optic nerve is widely regarded as a tract of the brain.

In 1981, Gross et al. ${ }^{10)}$ reported that frontal loading can result in the deformation of the ipsilateral orbital roof near the optic foramen, and that such deformation of the orbital roof could damage not only the supporting vasculature of the optic nerve, but could also cause shear stress to the nerve. This report is highly recognizable and frequently cited.

Following trauma, the optic nerve, particularly where it enters the optic canal, and retinal ganglion cell (RGC) axons are immediately sheared. Optic nerve swelling, particularly where the nerve enters the optic canal, follows due to vascular ischemia and direct mechanical trauma. This twostage model of TON became the basis for the treatment of TON. $^{27)}$

\section{Diagnosis of Indirect TON}

Diagnosis of indirect TON can be difficult because the initial ocular examination is typically normal, with the exception of an abnormal relative afferent pupillary defect (RAPD). Occasionally, the diagnosis of indirect TON can be delayed owing to severe head trauma. Furthermore, if both eyes are affected by optic neuropathy, the RAPD sign could be negative. Finally, head trauma, which leads to pupil dilation, can make the diagnosis of TON difficult.

TON has the following clinical features: 1) a positive RAPD sign, except in cases of bilateral ocular involvement; 2) impairment of color vision; and 3) visual field defects. ${ }^{27)}$ Visual acuity ranges from no light perception to normal. Optic atrophy develops after 4 to 6 weeks.

Optic nerve diseases lead to RGC degeneration, visual field loss, and potential blindness. With advanced diagnostic devices, Kanamori et al. ${ }^{12)}$ demonstrated that the reduc-
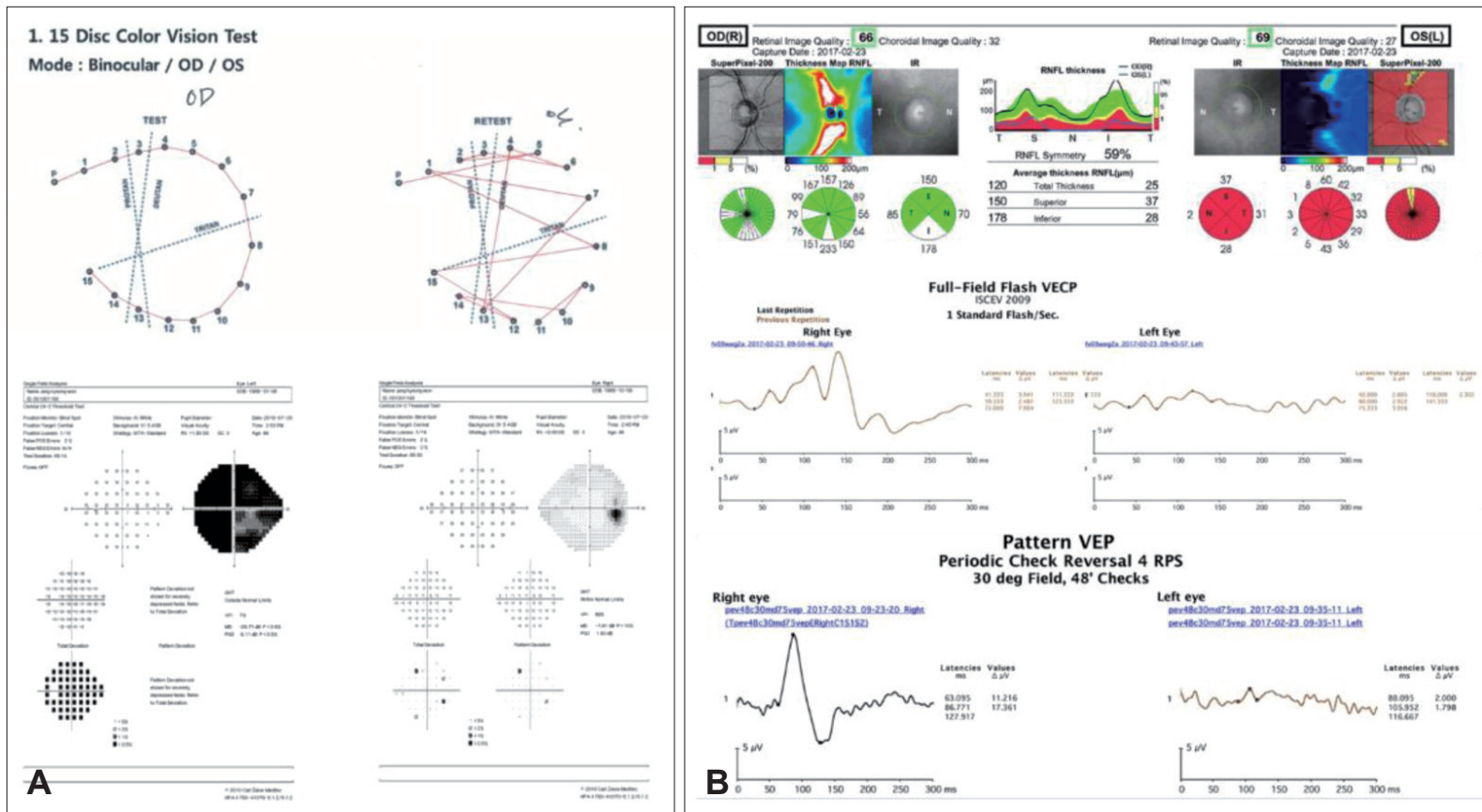

FIGURE 1. Representative case of forty-nine male who was diagnosed with left superior, lateral and inferior orbital wall fracture due to fall-down. (A) Color vision and visual field test result at 1 month after trauma. Note the impairment of color vision and total visual field constriction of left eye of the patient, (B) optical coherence tomography and visual evoked potential (VEP) test result at 18 months after trauma. Note the $360^{\circ}$ retinal nerve fiber layer thinning around the left disc and decreased response of left eye in VEP test. OD: right eye, OS: left eye, VECP: visually evoked cortical potential, ISCEV: the International Society for Clinical Electrophysiology of Vision. 
tions in circumpapillary retinal nerve fiber layer thickness and RGC complex began to decrease at 2 weeks after trauma and plateaued at 20 weeks in all cases. Figure 1 shows a representative case of typical indirect TON.

\section{Treatment of Indirect TON}

In 1982, in a report of seven cases of sudden monocular blindness following frontal head trauma, the following recommended indications were provided for optic nerve decompression: 1) delayed visual loss following frontal head trauma, unresponsive to 12 hours of megadose steroid therapy; and 2) initial return of vision with megadose steroids, followed by visual reduction while on steroids or with the tapering of steroid therapy. ${ }^{1)}$ In that report, the authors concluded that megadose steroids appeared to be useful in TON. Seiff ${ }^{22)}$ reported that $62 \%$ of treated patients and $33 \%$ of untreated patients showed vision improvement, in a study of 32 patients with indirect TON in 1990. Currently, the main treatment options for TON are 1) systemic steroid therapy; 2) optic nerve decompression; or 3) combined treatment of steroid therapy and optic nerve decompression. The management of indirect TON remains controversial. ${ }^{28-32)}$

The International Optic Nerve Trauma Study (IONTS) was first organized to investigate the optimal treatment of indirect TON. Accordingly, the study was initially conducted as a randomized controlled pilot study to compare 1) extracranial optic canal decompression combined with megadose corticosteroid; and 2) corticosteroid alone. However, due to the rarity of TON cases, randomized controlled trials (RCTs) could not be successfully conducted at that time. ${ }^{16)}$ Subsequently, IONTS group changed the study design to a comparative non-randomized interventional study and finally concluded that no clear benefit was found for either corticosteroid therapy or optic canal decompression surgery. A total of 133 patients with indirect TON were included in that study. ${ }^{16)}$ In 2011 and 2013, Cochrane reviews attempted to identify RCTs of TON in which any steroid regimens, either alone or in combination with surgical optic nerve decompression, were compared with surgery alone or with no treatment. ${ }^{30,31)}$ As a result, only one study was included: a double-masked, placebo-controlled, randomized trial of high-dose intravenous steroids in patients with indirect TON that was diagnosed within 7 days of the initial injury. ${ }^{8)}$ A total of 31 participants were randomly assigned to two groups (high-dose intravenous steroids group [ $n=16]$ or placebo group [n=15]). All participants were followed-up for three months. Mean final best-corrected visual acuity (BCVA) in the treatment group was $1.11 \pm 1.14$ and in the placebo group was $1.78 \pm 1.23$. This difference was not significant ( $p=0.13$ ). Visual acuity was improved in $68.8 \%$ of participants in the treatment group and $53.3 \%$ of participants in the placebo group, but the difference was not statistically significant $(p=0.38){ }^{8)}$ Thus, there has been no eligible study that could validate a particular strategy for the treatment of TON. However, in the ophthalmologic field, when TON is suspected, very high-dose steroid treatment should still be considered initially.

The rationale for megadose steroid therapy is based on the Second National Acute Spinal Cord Injury Study (NASCIS 2), which was a multicenter, randomized, double-blind, placebo-controlled study of patients with acute spinal cord injury. That study showed that treatment with methylprednisolone within 8 hours of injury resulted in a significant improvement in motor and sensory function, compared with placebo-treated patients. ${ }^{4)}$ The dosage of steroid was as follows: 1) low-dose (<100 mg); 2) moderate-dose (100-499 $\mathrm{mg}) ; 3$ ) high-dose (500-1,999 mg); 4) very high-dose (2,000$5,399 \mathrm{mg})$; and 5) megadose (>5,400 mg). Typically, the most commonly used steroid regimen in cases of TON is very high-dose intravenous methylprednisolone in the ophthalmologic field. ${ }^{27)}$ The spinal cord is a mixed grey and white matter tract of the brain, whereas the optic nerve is a pure white matter tract. The treatment success seen with methylprednisolone in the NASCIS 2 study may not be generalized to the treatment of TON. Furthermore, there might be other predisposing factors that could impact the effects of steroid treatment on indirect TON. Recently, Lai et al. ${ }^{15)}$ investigated predisposing factors for the recovery of patients with indirect TON who were treated with steroid pulse therapy. The authors commented that factors such as female sex, the administration of steroid therapy within less than 24 hours of the injury, lateral force fracture, and pure facial trauma revealed a better outcome for the improvement of visual acuity.

Since the treatment of TON is controversial, Emanuelli et al. ${ }^{7)}$ suggested their surgical and medical protocol for TON in 2015. The protocol consisted of a medial intravenous steroid treatment (within 8 hours of injury) and endoscopic surgical decompression (within 12-24 hours of the initiation of medical therapy). The authors reported that improvement of visual acuity was reached in $65 \%$ of cases, which is quite a high rate of successful treatment. ${ }^{7}$

A new treatment option for TON is the cytokine hormone erythropoietin (EPO). ${ }^{13,14)}$ EPO has been reported to be effective for reducing neural apoptosis and protecting brain ischemic injury. ${ }^{9}$ A pilot study of intravenous EPO for TON showed a promising result, in that patients in the EPO-treat- 
ed group showed a significantly better visual outcome than the observation group. ${ }^{13)}$ The same study group recently showed the effect of intravenous EPO for TON in an openlabel, phase 3, multicenter, semi-experimental trial. ${ }^{14)}$ All three groups (EPO, 69; steroid, 15; observation, ${ }^{16)}$ showed significant improvement of BCVA in patients with TON. Interestingly, color vision was significantly improved in the EPO group. The authors commented that no side effects were observed during follow up. ${ }^{14)}$

\section{Adverse Effect of Intravenous Steroid Treatment}

Lew et al ${ }^{17)}$ investigated the effect of high-dose steroid therapy in TON to detect changes in optic nerve head blood flow, concluding that the steroid therapy improved the optic nerve head blood flow, which might support the use of steroid treatment for indirect TON. If the benefits of a therapeutic intervention are unclear, clinicians should consider the risks of such intervention more carefully. The Optic Neuritis Treatment Trial used a high-dose steroid regimen: $250 \mathrm{mg}$ of intravenous methylprednisolone every 6 hours for 3 days, followed by $1 \mathrm{mg} / \mathrm{kg} /$ day of oral prednisolone for 11 days. Only two participants (1.3\%) in the intravenous methylprednisolone group had serious side effects: one case of acute psychosis and one case of acute pancreatitis, both resolving without sequelae. ${ }^{3)}$

The Corticosteroid Randomization after Significant Head Injury (CRASH) study, which was a large RCT investigating the effectiveness of steroid therapy in patients with acute traumatic brain injury, is well-known to have been terminated prematurely. ${ }^{6}$ In the CRASH study, patients with head trauma were allocated to either placebo or megadose intravenous methylprednisolone ( $2 \mathrm{~g} / \mathrm{h}$ for 1 hour, followed by $0.4 \mathrm{~g} / \mathrm{h}$ for 48 hours). The initial protocol was to recruit 20,000 participants, but the trial was terminated prematurely after 10,008 participants were enrolled. This decision was made by the data monitoring and ethics committee because the results showed that steroids were having a detrimental effect. At 6 months follow-up, the risk of death was higher in the steroid group than in the placebo group ( $25.7 \%$ vs. $22.3 \%$; relative risk [RR], $1.15 ; 95 \%$ confidence interval [CI], $1.07-1.24 ; p=0.0001)$, as was the risk of death or severe disability $(38.1 \%$ vs. $36.3 \%$; RR, 1.05 ; $95 \% \mathrm{CI}$, $0.99-1.10 ; p=0.079$ ). There was no evidence that the effect of steroids differed as a result of timing or severity of the injury.

\section{Conclusion}

In this review article, I surveyed the recent literature regarding TON and discussed how to manage indirect TON. Although most of the referenced papers were published many years ago, I have added new content through recently published papers in terms of classification, diagnosis, and new treatment options for TON.

Although the complications of steroid treatment are rare, there is no convincing evidence that steroid treatment provided any benefits in terms of improvement of visual acuity in patients with TON. Thus, patients with TON need to be made fully aware of both the theoretical risks suggested by recent studies and the practical risk of a serious side effect with active and aggressive intervention.

\section{Acknowledgement}

This study was supported by the National Research Foundation of Korea (NRF-2017R1A1A1A05001051), and the Soonchunhyang University Research Fund.

- The author has no financial conflicts of interest.

\section{REFERENCES}

1) Anderson RL, Panje WR, Gross CE. Optic nerve blindness following blunt forehead trauma. Ophthalmology 89:445-455, 1982

2) Ansari MH. Blindness after facial fractures: a 19 -year retrospective study. J Oral Maxillofac Surg 63:229-237, 2005

3) Beck RW, Cleary PA, Anderson MM Jr, Keltner JL, Shults WT, Kaufman DI, et al. A randomized, controlled trial of corticosteroids in the treatment of acute optic neuritis. The Optic Neuritis Study Group. N Engl J Med 326:581-588, 1992

4) Bracken MB, Shepard MJ, Collins WF, Holford TR, Young W, Baskin DS, et al. A randomized, controlled trial of methylprednisolone or naloxone in the treatment of acute spinal-cord injury. Results of the Second National Acute Spinal Cord Injury Study. N Engl J Med 322:1405-1411, 1990

5) Dancey A, Perry M, Silva DC. Blindness after blunt facial trauma: are there any clinical clues to early recognition? J Trauma 58:328335,2005

6) Edwards P, Arango M, Balica L, Cottingham R, El-Sayed H, Farrell $\mathrm{B}$, et al. Final results of MRC CRASH, a randomised placebocontrolled trial of intravenous corticosteroid in adults with head injury-outcomes at 6 months. Lancet 365:1957-1959, 2005

7) Emanuelli E, Bignami M, Digilio E, Fusetti S, Volo T, Castelnuovo P. Post-traumatic optic neuropathy: our surgical and medical protocol. Eur Arch Otorhinolaryngol 272:3301-3309, 2015

8) Entezari M, Rajavi Z, Sedighi N, Daftarian N, Sanagoo M. Highdose intravenous methylprednisolone in recent traumatic optic neuropathy; a randomized double-masked placebo-controlled clinical trial. Graefes Arch Clin Exp Ophthalmol 245:1267-1271, 2007

9) Ghezzi P, Brines M. Erythropoietin as an antiapoptotic, tissueprotective cytokine. Cell Death Differ 11 Suppl 1:S37-S44, 2004

10) Gross CE, DeKock JR, Panje WR, Hershkowitz N, Newman J. Evidence for orbital deformation that may contribute to monocular blindness following minor frontal head trauma. J Neurosurg 55:963-966, 1981

11) Hughes B. Indirect injury of the optic nerves and chiasma. Bull 
Johns Hopkins Hosp 111:98-126, 1962

12) Kanamori A, Nakamura M, Yamada $Y$, Negi A. Longitudinal study of retinal nerve fiber layer thickness and ganglion cell complex in traumatic optic neuropathy. Arch Ophthalmol 130:1067-1069, 2012

13) Kashkouli MB, Pakdel F, Sanjari MS, Haghighi A, Nojomi M, Homaee $\mathrm{MH}$, et al. Erythropoietin: a novel treatment for traumatic optic neuropathy-a pilot study. Graefes Arch Clin Exp Ophthalmol 249:731-736, 2011

14) Kashkouli MB, Yousefi S, Nojomi M, Sanjari MS, Pakdel F, Entezari M, et al. Traumatic optic neuropathy treatment trial (TONTT): open label, phase 3, multicenter, semi-experimental trial. Graefes Arch Clin Exp Ophthalmol 256:209-218, 2018

15) Lai IL, Liao HT, Chen CT. Risk factors analysis for the outcome of indirect traumatic optic neuropathy with steroid pulse therapy. Ann Plast Surg 76 Suppl 1:S60-S67, 2016

16) Levin LA, Beck RW, Joseph MP, Seiff S, Kraker R. The treatment of traumatic optic neuropathy: the International Optic Nerve Trauma Study. Ophthalmology 106:1268-1277, 1999

17) Lew H, Lee SY, Jang JW, Kim HY, Kang SJ, Kim SJ. The effects of high-dose corticosteroid therapy on optic nerve head blood flow in experimental traumatic optic neuropathy. Ophthalmic Res 31: 463-470, 1999

18) Mackiewicz J, Tomaszewska J, Jasielska M. Optic nerve avulsion after blunt ocular trauma - Case report. Ann Agric Environ Med 23:382-383, 2016

19) Magarakis M, Mundinger GS, Kelamis JA, Dorafshar AH, Bojovic $\mathrm{B}$, Rodriguez ED. Ocular injury, visual impairment, and blindness associated with facial fractures: a systematic literature review. Plast Reconstr Surg 129:227-233, 2012

20) Sarkies N. Traumatic optic neuropathy. Eye (Lond) 18:1122-1125,
2004

21) Sawhney R, Kochhar S, Gupta R, Jain R, Sood S. Traumatic optic nerve avulsion: role of ultrasonography. Eye (Lond) 17:667-670, 2003

22) Seiff SR. High dose corticosteroids for treatment of vision loss due to indirect injury to the optic nerve. Ophthalmic Surg 21:389395,1990

23) Sosin M, De La Cruz C, Mundinger GS, Saadat SY, Nam AJ, Manson PN, et al. Treatment outcomes following traumatic optic neuropathy. Plast Reconstr Surg 137:231-238, 2016

24) Spoor TC, Hartel WC, Lensink DB, Wilkinson MJ. Treatment of traumatic optic neuropathy with corticosteroids. Am J Ophthalmol 110:665-669, 1990

25) Steinsapir KD, Goldberg RA. Traumatic optic neuropathy. Surv Ophthalmol 38:487-518, 1994

26) Xie D, Yu H, Ju J, Zhang L. The outcome of endoscopic optic nerve decompression for bilateral traumatic optic neuropathy. J Craniofac Surg 28:1024-1026, 2017

27) Yu-Wai-Man P. Traumatic optic neuropathy-Clinical features and management issues. Taiwan J Ophthalmol 5:3-8, 2015

28) Yu-Wai-Man P, Griffiths PG. Surgery for traumatic optic neuropathy. Cochrane Database Syst Rev:CD005024, 2005

29) Yu-Wai-Man P, Griffiths PG. Steroids for traumatic optic neuropathy. Cochrane Database Syst Rev:CD006032, 2007

30) Yu-Wai-Man P, Griffiths PG. Steroids for traumatic optic neuropathy. Cochrane Database Syst Rev:CD006032, 2011

31) Yu-Wai-Man P, Griffiths PG. Steroids for traumatic optic neuropathy. Cochrane Database Syst Rev:CD006032, 2013

32) Yu-Wai-Man P, Griffiths PG. Surgery for traumatic optic neuropathy. Cochrane Database Syst Rev 6:CD005024, 2013 